\section{Skilled eyes are needed to go on studying the richness of the soil}

Sir-Many ecologists are unaware that most of the biodiversity in any terrestrial ecosystem occurs in the soil, so we were delighted to see your recent News Feature ${ }^{1}$ We do not agree, however, with the implication that such studies have been initiated only recently: in fact, the biodiversity and functioning of soil organism communities have been a major research area for soil ecologists for 50 years.

As early as 1950, Kühnelt ${ }^{2}$ stressed the diversity of soil dwellers. A wave of publications outlining soil organism diversity occurred in the 1970s, including the recognition of the 'enigma' of high species diversity associated with apparent high trophic overlap ${ }^{3}$. The importance of soil organisms for plant growth has also been recognized for more than a century. Currently, soil biodiversity is even being seen as a tool for sustainable agriculture ${ }^{4}$.

Progress in soil ecological research has often been constrained by taxonomic and methodological problems, which are not resolved in some of the studies cited in your News Feature, making it difficult to reach meaningful levels of taxonomic resolution. Experimental manipulations such as removing starfish from a stretch of rocky shore are said to be extremely difficult to apply to soil-dwellers. Yet manipulative studies have been carried out with soil organisms for many years. These involve many different types of experimental approach including the use of litter-bags with different mesh sizes, partial or complete defaunation using pesticides, transplantation of soils and inoculation of experimental soil units. These experiments achieved in the field during the past 20 years or more, however difficult they are to perform, have been instructive in supplementing laboratory results and providing foundations for the studies cited ${ }^{1}$.

The canopy has been called the 'last biotic frontier of the biosphere $e^{5}$, and perhaps the soil can join it ${ }^{6}$. Unfortunately, funds for the study of nematodes, mites and other minute soil animals disappear into the ravening maw of molecular biology. If there is no one left who can tell one soil animal from another, however, we will be exploring these frontiers blind. Henri M. André ${ }^{\star} \dagger$, Xavier Ducarme $\dagger$, Jo M. Anderson $\$$, David A. Crossley Jrll, Hartmut H. Koehlerg, Maurizio G. Paoletti\#, David E. Walter $\downarrow$, Philippe Lebrun $\dagger$ ${ }^{*}$ UR Faune du sol, Musée royal de l'Afrique centrale, B-3080 Tervuren, Belgium †Unité d'Écologie et de Biogéographie, Université catholique de Louvain, Place Croix du Sud 4/5,
B-1348 Louvain-la-Neuve, Belgium $\ddagger$ Aspirant au Fonds national de la Recherche scientifique, Belgium

\$School of Biological Sciences, University of Exeter, Exeter EX4 4PS, UK

IIInstitute of Ecology, Ecology Annex, University of Georgia, Athens, Georgia 30602-2360, USA

IUniversität Bremen FB2, Institute of Ecology and Evolutionary Biology, Centre of Environmental

Research \& Environmental Technology,

Leobenerstr., PO Box 330 440, D-28359 Bremen, Germany

\#Dipartimento di Biologia, Università di Padova, via U. Bassi, 58/b, I-35121 Padova, Italy

\&Department of Zoology \& Entomology, HartleyTeakle Building, University of Queensland, St Lucia, Queensland 4072, Australia

1. Copley, J. Nature 406, 452-454 (2000).

2. Kühnelt, W. Bodenbiologie (Herold, Vienna, 1950).

3. Anderson, J. M. in Progress in Soil Zoology (ed. Vanek, J.) 51-58 (Academia, Prague, 1975)

4. Paoletti, M. G. Agriculture, Ecosystems \& Environment 74, 1-18 (1999).

5. Erwin, T. L. Bull. Ent. Soc. Amer. 29, 14-19 (1983).

6. André, H. M., Noti, M. I. \& Lebrun, P. Biodivers. Conserv. 3, 45-56 (1994).

\section{Astronomy network will allow every site to shine}

Sir - The News Feature on optical telescopes (Nature 408, 12; 2000) reviews the present state of most national telescope operators in Europe and the United States, and identifies some important niches that medium-sized (2-4-m class) telescopes will fill in the future. The story seems to suggest, however, that it is necessary to close most nationally operated 2-4-m-class telescopes in Europe and focus operation on a single site. This is not what has been discussed recently.

What is required is that national telescope operators establish a single European network to coordinate operations of the observatory facilities and the instrumentation of their telescopes. This will allow each site to focus on its particular strengths, while assuring a comprehensive instrumentation complement across the network. The coordination will introduce synergies and allow more efficient operation at each site, which in turn will reduce costs. Without such coordination among national facilities, individual telescopes might no longer achieve the funding to remain competitive and might close prematurely.

At the same time, the network should offer time to European countries that do not operate national observatories or which have no general access to international observatories such as the European Southern Observatory. Access to other European facilities may foster astronomy programmes in these countries, in the way that Spanish astronomy has benefited from access to installations such as the Institute of Millimetric Radioastronomy in the French Alps, the Isaac Newton Group (ING) on La Palma in the Canary Islands and Calar Alto on mainland Spain.

There has been a very large response to the creation of such a network. During a recent meeting of the Optical and Infrared Coordination Network for Astronomy (OPTICON), members agreed to study a system of instrument coordination and development, and to consider systems to extend telescope access, exchange time between telescopes, and enhance the education and training role of its facilities.

The expected synergies are illustrated by ING and the Calar Alto observatory. With Omega2000, Calar Alto will be operating a near-infrared camera that provides the largest field of view at any telescope in the northern hemisphere. On La Palma, on the other hand, instrumentation efforts focus on adaptive optics and wide-field spectroscopy. Access to both wide-field imaging and wide-field spectroscopy at two different sites offers obvious benefits to the broader European community.

Therefore, medium-sized telescopes can have an exciting future. The undersigned participate as coordinators of two OPTICON working groups which aim to implement the ideas described here. Roland Gredel ${ }^{\star}$, Rene Rutten $\dagger$ ${ }^{*}$ Calar Alto Observatory, Jesus Durban 2,2, Apartado de Correos 511, E-04080 Almeria, Spain †Isaac Newton Group of Telescopes, Apartado de Correos 321, 38700 Santa Cruz de La Palma, Islas Canarias, Spain

\section{Semmelweis and the battle against infection}

Sir-Fred Rosen's review of The War Within Us by Cedric Mims (Nature 408, 769; 2000) mentions Semmelweis, who introduced antisepsis into medical practice. Born in Budapest in 1818 to a family of German descent, he was called Ignaz Philipp in German and Ignác Fülöp in Hungarian. He considered himself Hungarian and attended medical school in Hungary, but pursued some of his professional career in Vienna where he encountered both success in his work, and humiliation by his jealous boss.

Being male, however, he did not exactly die from "the very disease he spent his life trying to eradicate" - since this was puerperism, or childbed fever. Rather, he died of staphylococcal infection, the agent that is most often responsible for post-partum death.

George Rédei

3005 Woodbine Court, Columbia, Missouri 65203-0906, USA 